Uwe Geith* und Thomas Kraß

\title{
Open Access im Blick! Mehr Sichtbarkeit von Open-Access-Publikationen in der Bibliothek - Projekt visOA
}

https://doi.org/10.1515/bfp-2020-2088

Zusammenfassung: Um Open-Access-Publikationen stärker in den Wahrnehmungsbereich der Bibliotheksbenutzer $\mathrm{zu}$ bringen, ist es notwendig, sie besser sichtbar zu machen. Im visOA-Projekt, das von der NTNM-Bibliothek des INM durchgeführt wurde, wurden Open-Access-Publikationen sowohl im OPAC als auch im Discovery-System und in den Publikationslisten des INM deutlich hervorgehoben. Eine selbstprogrammierte virtuelle Zeitschriftenanzeige (VIDIJO) ${ }^{1}$ bietet eine fachspezifische Auswahl von Open-Access-Zeitschriften. Die Wirksamkeit der im Projekt ergriffenen Maßnahmen wurde durch Begleitforschung überprüft.

Schlüsselwörter: Open Access; Visualisierung; virtuelle Zeitschriftenauslage

\section{Open Access at a Glance! More Visibility of Open Access Publications in the Library - The visOA Project}

Abstract: In order to bring Open Access publications more into the perception of library users, it is necessary to make them more visible. In the visOA project, carried out by the NTNM library of the INM, Open Access publications were clearly highlighted in the OPAC as well as in the Discovery System and in the publication lists of the INM. A self-programmed virtual journal display (VIDIJO) also offers a subject-specific selection of Open Access journals. The effectiveness of the measures taken in the project was verified by accompanying research.

Keywords: Open Access; visualization; virtual journal display

\section{Einleitung}

Das INM - Leibniz-Institut für Neue Materialien ist ein internationales Zentrum für Materialforschung mit Sitz in Saarbrücken. Es vereint multidisziplinäre Wissenschaft und materialorientierten Technologietransfer unter einem Dach. Chemie, Physik, Biologie, Materialwissenschaft und Engineering wirken in enger Kooperation auf hohem Niveau zusammen. Ein wesentlicher Fokus seiner Arbeit ist die Übertragung von biologischen Prinzipien auf das Design neuer Materialien, Strukturen und Oberflächen. Die NTNM-Bibliothek ist die gemeinsame Bibliothek des INM Leibniz-Institut für Neue Materialien (INM) und der Naturwissenschaftlich-Technischen Fakultät (NT) der Universität des Saarlandes.

Der Open-Access-Beauftragte des INM - Leibniz-Institut für Neue Materialien, ein Mitarbeiter der NTNM-Bibliothek, beteiligte sich erfolgreich an einem Ideenwettbewerb im Rahmen einer Förderrichtlinie des Bundesministeriums für Bildung und Forschung (BMBF). Ziel des vom Bundesministerium für Bildung und Forschung (BMBF) geförderten Projekts ${ }^{2}$ Sichtbarmachung von Open-Access-Publikationen in den Nachweisinstrumenten einer Bibliothek visOA war es, die Sichtbarkeit von Open-Access-Publikationen zu verbessern, indem sie deutlich gekennzeichnet und hervorgehoben werden. Durch die stärkere Sichtbarmachung von Open-Access-Publikationen soll die Wahrnehmung und Bekanntheit des Themas Open Access bei Publizierenden und Rezipienten erhöht werden. Dadurch könnte die Etablierung von Open Access beschleunigt werden.
1 https://www.vidijo.org/journals.

*Kontaktperson: Uwe Geith, uwe.geith@leibniz-inm.de Thomas Kraß, krass@ntnm-bib.de
2 Die Förderung erfolgte im Rahmen der „Förderrichtlinie des freien Informationsflusses in der Wissenschaft - Open Access des Bundesministeriums für Bildung und Forschung (BMBF)" mit dem Förderkennzeichen: 160A060. Der Förderzeitraum erstreckte sich vom 01.03.2018 bis zum 31.12.2019. 
10. Choosing the right carbon additive is of vitalimportance for high-performance Sb-based Na-ionbatteries / Chhristina Pfeifer, Stefanie Arnold, Oznil Budak, Xianlin Luo, Volker Presser, Helmut Ehrenberg and Sonia Dsoke

von Pfeifer, Kristina [Verfasserin] | Arnold, Stefanie [VerfasserIn] | Budak, Öznil [Verfasserln] | Luo, Xianlin [VerfasserIn] | Presser, Volker [VerfasserIn] | Ehrenberg, Helmut [Verfasserin] | Dsoke, Sonia [VerfasserIn] I Leibniz-Institut fur Neue Materialien [Sonstige Person, Familie und Korperschaft]

Quelle: Joumal of materials chemistry /A

Materialtyp: $\square$ Aufsatz; Format online verügbar 3

Verlag: 2020

Online-Ressourcen: Kostentrei

Verfugbarkeit: Ausleihbare Exemplare: NTNM-Bibliothek [Signatur: INM 2020/035] (1)

Listen: INM-Publikationen 04_2020.

a Vormerken Meinem Korb hinzufügen

11. AVENUE21. Automatisierter und vernetzter Verkehr: Entwicklungen des urbanen Europa / von Mathias Mitteregger, Emilia M. Bruck, Aggelos Soteropoulos, Andrea Stickler, Martin Berger, Jens S. Dangschat, Rudolf Scheuvens, lan Banerjee

von Mitteregger, Mathias [Verfasserln] | Bruck, Emilia M [Verfasserln] | Soteropoulos, Aggelos [Verfasserln] | Stickler, Andrea [Verfasserln] | Berger, Martin [Verfasserln] | Dangschat, Jen S Nerfasserin] | Scheuvens, Rudolf [Verfasserin] | Banerjee, lan [Verfasserin]

Auflage: 1st ed. 2020

Materialtyp: 1 Buch; Format: online verfugbar 3

Verlag. Berlin, Heidelberg : Springer Berlin Heidelberg. 2020

Verlag: Berlin, Heidelberg : Imprint: Springer Vieweg. 2020

Online-Ressourcen: Zugriff nur aus dem Netz der Universitatat des Saarlandes, kein Volltext-Zugriff aus dem IP-Bereich des INM

yeinem Korb hinzufügen

12. Dual-zinc electrode electrochemical desalination / Jinhong Dai, Jian Wang, Xianhua Hou, Qiang Ru, Qingyu He, Pattarachai Srimuk, Volker Presser, and Fuming Chen

von Dai, Jinhong [Verfasserln] | Wang, Jian [Verfasserln] | Hou, Xianhua [Verfasserin] | Ru, Qiang [Verfasserln] | He, Qingyu [Verfasserln] | Srimuk, Pattarachai [Verfasserln] | Presser, Volker [Verfasserln] | Chen, Fuming [VerfasserIn] | Leibniz-Institut für Neue Materialien [Sonstige Person, Familie und Körperschaft].

Quelle: ChemSusChem

Materialtyp: $\square$ Aufsatz; Format: online verugigbar 3

Verlag: 2020

Online-Ressourcen: Kostentrei

Verfugbarkeit: Ausleihbare Exemplare: NTNM-Bibliothek [Signatur. INM 2020/039] (1).

Listen: INM-Publikationen $04 \_2020$

a Vormerken Meinem Korb hinzufügen

Abb. 1: Open-Access-Publikationen im NTNM-OPAC

\section{Maßnahmen}

Im Projekt visOA wurden folgende Maßnahmen umgesetzt:

- Fachliche Selektion von Open-Access-Publikationen für und deren Aufnahme in den Online-Katalog.

- Deutliche Kennzeichnung von Open-Access-Publikationen im Online-Katalog.

- Deutliche Kennzeichnung von OA-Publikationen im NTNM-Wissensportal (einem Discovery System).

- Deutliche Kennzeichnung von Open-Access-Publikationen in der Publikationsdatenbank und den Publikationslisten des INM - Leibniz-Instituts für Neue Materialien.

- Erstellung einer „virtuellen Zeitschriftenauslage“ für fachlich ausgewählte Open-Access-Journals.

- Begleitende Forschung über die Wirksamkeit der Maßnahmen.

Die Recherche nach fachlich interessanten OA-Publikationen erfolgte in der Datenbank des Südwestdeutschen Bibliotheksverbundes Baden-Württemberg, Saarland, Sachsen (SWB, jetzt Teil von K10plus) und im Directory of Open Access Journals (DOAJ). Im SWB sind online frei zugängliche Dokumente als lizenzfrei gekennzeichnet und unter dem virtuellen Bibliothekssigel LFER $^{3}$ zusammengefasst. Die Übernahme in den lokalen OPAC erfolgt nach Anlegen eines Lokalsatzes für die NTNM-Bibliothek automatisch. Mehr Aufwand machen Treffer aus dem DOAJ. Diese müssen meist erst noch im SWB erfasst werden.

Im OPAC der NTNM-Bibliothek werden nun alle als lizenzfrei gekennzeichneten Publikationen bei der Anzeige visuell hervorgehoben: In den Trefferlisten einer Recherche durch farbliche Kennzeichnung des jeweiligen Treffers und durch die Anzeige eines Open-Access-Logos dort, wo sonst ein Coverbild erscheint. Bei der detaillierten Einzelanzeige eines Treffers geschieht die Kennzeichnung ebenfalls durch ein großes Open-Access-Logo.

Im NTNM-Wissensportal ${ }^{4}$ (einem EDS von EBSCO) werden Open-Access-Publikationen ebenfalls durch ein Open-Access-Logo gekennzeichnet. Ist der Treffer im Index des EDS eindeutig als Open-Access-Publikation zu erkennen, indem er z.B. aus der Datenbank des DOAJ stammt, wird das entsprechende Icon des Medientyps

3 https://swb.bsz-bw.de/DB=2.256.

4 OPAC und Wissensportal der NTNM-Bibliothek sind über zwei Suchschlitze auf der Startseite der Webseite (https://www.ntnm-bib. de) recherchierbar. Der OPAC (NTNM-Katalog) ist auch unter https://i nm.bsz-bw.de erreichbar. 
durch ein Open-Access-Logo überlagert. Durch die Nutzung der Unpaywall-App wird darüber hinaus beim Aufbau von Trefferlisten im Hintergrund ermittelt, ob es von einem Treffer eine Open-Access-Version der Publikation gibt. Ist dies der Fall, wird bei den weiterführenden Links auch ein Link zur Open-Access-Version angegeben, dem ein allerdings viel kleineres Open-Access-Logo vorangestellt ist.

\section{Wissenschaftliche Begleitforschung}

Um die Wirksamkeit der im Projekt realisierten Maßnahmen zur verbesserten Sichtbarmachung von Open Access in den Nachweisinstrumenten der NTNM-Bibliothek zu überprüfen, wurde durch einen externen Dienstleister ${ }^{5}$ Begleitforschung zur qualitativen Wahrnehmung von Open Access durch Wissenschaftler vorgenommen. Diese umfasste verschiedene Erhebungen: eine Literaturstudie, qualitative Interviews, eine Online-Umfrage zu zwei Erhebungszeitpunkten.

Die von Dr. Ulrich Herb durchgeführte Literaturstudie ${ }^{6}$ fokussierte auf die inhaltlichen Faktoren, welche die Wahrnehmung der Qualität einer Publikation beeinflussen. Basierend auf dieser Erhebung wurde ein Kriterienkatalog zur qualitativen Bewertung wissenschaftlicher Publikationen entwickelt. Dieser diente wiederum der Erstellung von Bögen für Leitfadeninterviews, ${ }^{7}$ in denen geprüft wurde, inwiefern die in der Literatur berichteten Faktoren im fachlichen Kontext von visOA tatsächlich Einfluss auf die qualitative Wahrnehmung wissenschaftlicher Werke haben. Die Resultate dieser Leitfadeninterviews gingen in die Entwicklung einer Online-Umfrage ein, die ermitteln sollte, wie die qualitative Wahrnehmung von Open-Access- und Closed-Access-Publikationen unter den Wissenschaftlern des INM ausfällt und ob und wie sie sich im Laufe des Projekts und der darin getroffenen Maßnahmen änderte. ${ }^{8}$

Nach Durchführung der zweiten Online-Umfrage zum Projektende hin und der Auswertung beider Umfragen konnte unter anderem festgestellt werden, dass trotz stär-

5 Dr. Ulrich Herb /scidecode science consulting \& research.

6 Ulrich Herb (2019a).

7 Ulrich Herb (2019b).

8 Über die Literaturstudie, den Kriterienkatalog und die qualitativen Interviews mit Wissenschaftlern über die Wahrnehmung der Qualität von wissenschaftlichen Publikationen gibt Herb und Geith (2020) Auskunft. kerer Wahrnehmung durch die getroffenen Maßnahmen sich die qualitative Wahrnehmung von Open-Access-Publikationen nicht signifikant verändert hat. ${ }^{9}$

\section{Die virtuelle Zeitschriftenauslage VIDIJO}

Besonders arbeitsintensiv war die Konzeption und Programmierung einer „virtuellen Zeitschriftenauslage“ speziell für Open-Access-Journals, genannt VIDIJO (Abkürzung für „virtual display of journals“). Dabei sollte der Weg der Informationsbeschaffung, wie er typischerweise bei einer physischen Zeitschriftenauslage für Print-Zeitschriften verläuft, auch im virtuellen Zeitschriftenangebot realisiert werden. Über eine Browsing-Ansicht, die visuell die physische Zeitschriftenauslage in der Bibliothek nachbildet, gelangt man durch Anklicken eines ZeitschriftenCovers zum Inhaltsverzeichnis der jeweiligen Zeitschrift; alle in diesem Journal publizierten Artikel werden dort als sortierte Liste aufgeführt, so dass die neuesten Artikel zuerst erscheinen. Durch Anklicken eines Listeneintrags wird das Element ausgeklappt und weitere Informationen wie weitere Autoren, das Publikationsdatum und der Abstract des Artikels sowie seine DOI werden sichtbar. Über den Button „Open Article“ gelangt man direkt zum gewünschten Artikel.

Da VIDIJO nur Open-Access-Journals enthält, unterliegen die Publikationen keiner Beschränkung auf einen bestimmten lizensierten IP-Bereich und sind deshalb von überall nutzbar: nicht nur in der NTNM-Bibliothek, am INM und/oder an der Universität des Saarlandes, sondern auch von zuhause oder auf Reisen - von überall, wo ein Internet-Zugang möglich ist.

Durch Verwendung moderner Web-Technologien wird es in einer geplanten Erweiterung sogar möglich sein, innerhalb einer Sitzung mit bereits abgerufenen Daten weiterzuarbeiten, falls die Internetverbindung unterbrochen werden sollte (z. B. auf Zugfahrten). Nach Wiederherstellung der Verbindung werden die Daten synchronisiert.

\subsection{Konzept}

Bei der Konzeption der virtuellen Zeitschriftenauslage spielten auch die früheren Erfahrungen des Projektleiters

9 Einzelheiten über die beiden Online-Umfragen und deren Ergebnisse werden im Juli 2020 auf der Projekt-Webseite veröffentlicht. 


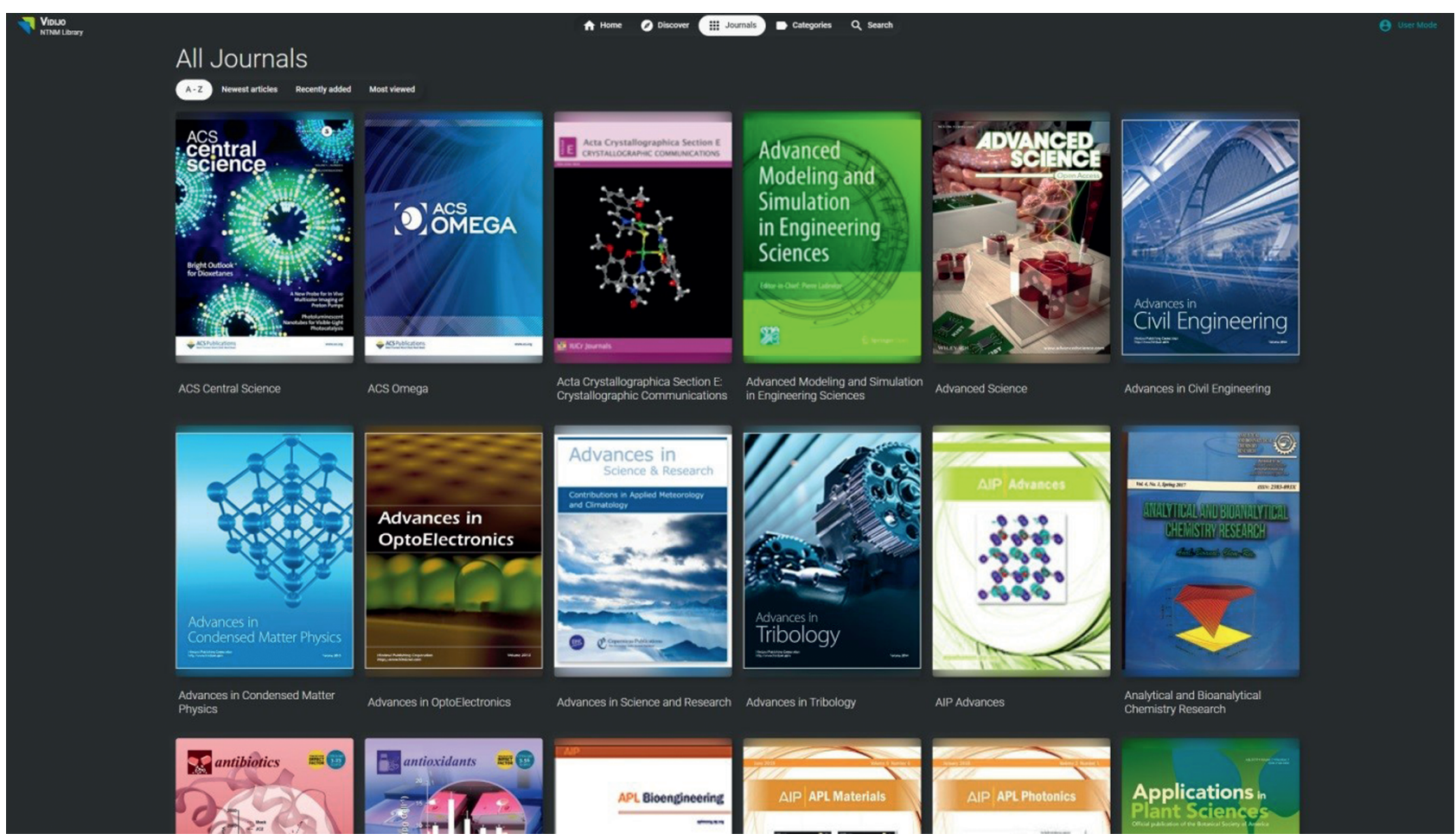

Abb. 2: Die virtuelle Zeitschriftenauslage der NTNM-Bibliothek

mit dem Thema „Visualisierungen in Bibliotheken“ eine Rolle. Als erstes ist das Projekt Blended Library ${ }^{10}$ zu nennen, insbesondere das im Rahmen dieses Projekts entstandene Blended Shelf. ${ }^{11}$ Einer der Grundgedanken der Blended Library ist die Verbindung von physischem und virtuellem Raum in der Bibliothek. Die ersten Versionen des Blended Shelf sind ein Ergebnis der Masterarbeit von Eike Kleiner ${ }^{12}$ und boten eine 3D-Visualisierung von Bibliotheksbeständen (sowohl den Printbestand als auch EBooks) in der Form von virtuellen Bibliotheksregalen.

Auch die Idee einer virtuellen Zeitschriftenauslage ist nicht neu: Mit dem Tool JournalTouch ${ }^{13}$ versuchte schon 2015 die Bibliothek des Max-Planck-Instituts zur Erforschung von Gemeinschaftsgütern die analoge Zeitschriftenauslage in die digitale Welt zu übertragen. Da das INM nach Realisierung der NTNM-Bibliothek am anderen Ende des Saarbrücker Campus keine Bibliothek mehr im eigenen Haus hatte, versuchten wir dies durch eine JournalTouch-Installation mit dem Zeitschriftenbestand der NTNM-Bibliothek, die auf Touchbildschirmen nicht nur in der Bibliothek, sondern auch im INM zugänglich sein soll- te, etwas $\mathrm{zu}$ kompensieren. Leider erlosch das Interesse der Nutzer an diesem neuen Tool bei einer mehrwöchigen Teststellung schon nach recht kurzer Zeit und so wurde der Service nicht verwirklicht. Aus dem Feedback der Nutzer zu JournalTouch, den Erfahrungen von Nutzern des Blended Shelfs und aus einem Vergleich unterschiedlicher Visualisierungstools für Zeitschriften ${ }^{14}$ versuchten wir, im Projekt visOA zu lernen und die von uns neu geschaffene virtuelle Zeitschriftenauslage VIDIJO sehr viel stärker an den Bedürfnissen unserer Nutzer auszurichten. Wir wollten unseren Nutzern mit VIDIJO also ein Tool zur Verfügung stellen, das nicht nur seine Nutzer mit den vielen Open-Access-Zeitschriften in seinen Fachbereich bekannt machen und als Browsing-Instrument die Möglichkeit zur Serendipität bieten sollte. Dieses Tool sollten sie darüber hinaus überall nutzen können und es sollte ihnen unmittelbaren Zugang zu Publikationen bieten. Gleichzeitig sollte das Interface so gestaltet sein, dass es sich von selbst erklärt, indem es einerseits bekannte Vorgehensweisen zur Zeitschriftennutzung abbildet und andererseits in Gestaltung und Bedienung mit vielen anderen, bereits in anderem Zusammenhang bekannten Applikationen übereinstimmt. Wichtig war uns dabei die konsequente Aus-

10 Reiterer et al. (2016).

11 Kleiner et al. (2013).

12 Kleiner (2014).

13 Goldschmitt (2016).

14 Patel (2019). 
richtung an den Bedürfnissen und Erwartungen der Nutzer. $^{15}$

\subsection{Realisierung}

Um die Anforderungen an VIDIJO erfolgreich umsetzen zu können, wurde auf die Verwendung des ca. 20 Jahre alten LAMP-Stacks zu Gunsten des moderneren MEAN-Stacks verzichtet.

Der MEAN-Stack besteht aus den folgenden Komponenten:

- die NoSQL-Datenbank MongoDB,

- das serverseitige Webapplikations-Framework Express.js,

- das clientseitige Frontend-Framework Angular

- und die auf der JavaScript-Laufzeitumgebung aufbauende Plattform Node.js.

Die Notwendigkeit verschiedener Programmiersprachen für Client (JavaScript) und Server (PHP etc.) wird durch Verwenden dieser Software-Auswahl hinfällig, so dass TypeScript als einzige Programmiersprache im gesamten Projekt verwendet werden konnte. Dies ermöglicht eine vereinfachte Wiederverwendung von eigenem Code und Drittanbieter-Paketen und somit eine beschleunigte Entwicklung von VIDIJO.

Das funktionsreiche Frontend-Framework „Angular“ macht es möglich, VIDIJO als Single Page Application umzusetzen (das heißt, dass die Webseite nur einmal geladen werden muss und Inhalte dynamisch nachgeladen werden). Vor allem neu hinzugefügte Journals und kürzlich publizierte Artikel können so dynamisch in die Applikation geladen werden. Will man VIDIJO nicht wie eine Webseite verwenden, so kann es über den Browser installiert und anschließend als sogenannte „Progressive Web App“ benutzt werden; dies funktioniert sowohl auf Mobilgeräten, als auch auf Desktops - wie nach der Installation normaler Apps kann VIDIJO anschließend vom App-Starter aus geöffnet werden.

VIDIJO wird über die Containervirtualisierungssoftware „Docker“ betrieben. Damit wird sichergestellt, dass das Projekt auf jedem Server gleichermaßen betrieben werden kann, unabhängig vom eingesetzten Betriebssystem. Weitere Vorteile sind eine gute Skalierbarkeit mit

15 Häufig werden neue Tools so konzipiert, wie Bibliothekare sich denken, was Nutzer bräuchten und wie sie Angebote nutzen sollten. Eine strikte Ausrichtung an den Bedürfnissen und Vorgehensweisen der Nutzer verspricht allerdings mehr Erfolg und führt zu höherer Kundenzufriedenheit.
Anstieg der Nutzerzahlen sowie automatisches Neustarten der einzelnen Komponenten bei Ausfällen.

Neben der Browsing-Ansicht gibt es auch Einstiege nach Kategorien bzw. Fachgebieten und eine allgemeine „Discovery“-Ansicht mit den neuesten Artikeln, mit den neu hinzugekommen und den meistgenutzten Journals.

Außerdem stehen nach der Erstellung eines persönlichen Accounts weitere Funktionen zur Verfügung:

- Man kann bei den Journals Favoriten bestimmen.

- Man kann interessante Artikel auf eine persönliche Leseliste setzen.

- Auf der individuell angepassten „HOME“-Seite werden die neuesten Artikel favorisierter Zeitschriften, die persönliche Leseliste und die Journal-Favoriten aufgeführt.

- Man kann also VIDIJO ganz nach seinen persönlichen Bedürfnissen und Vorlieben einrichten.

Für den Einsatz von VIDIJO ergaben sich in der NTNMBibliothek verschiedene Möglichkeiten. So gibt es für VIDIJO einerseits einen Touch-Bildschirm im Eingangsbereich der NTNM-Bibliothek, der jedoch nur als Eye-Catcher und Tool zur ersten „Kontaktaufnahme“ mit unserem Angebot dient. Auf den Arbeitsplatz-PCs in der Bibliothek wird neben anderen nützlichen Programmen auch eine prominent platzierte Desktop-Verknüpfung zum Aufruf von VIDIJO angeboten. Konzipiert und realisiert ist VIDIJO aber in erster Linie als überall nutzbare Anwendung (auf einem privaten Desktop-PC, Notebook, Tablet oder Smartphone etc.).

\section{Nachnutzung}

Besonderen Wert wurde in allen vom BMBF geförderten Projekten auf Nachhaltigkeit der Projekte gelegt. Im Projekt visOA sollten die einzelnen Maßnahmen deshalb nachhaltig sein. Die Nachhaltigkeit sehen wir dadurch gewährleistet, dass die einzelnen Maßnahmen für andere Bibliotheken und Informationseinrichtungen nachvollziehbar und in deren Nachweis-Instrumenten nachnutzbar sind. Die virtuelle Zeitschriftenauslage VIDIJO ist nach Fertigstellung der stabilen Version VIDIJO 1.0.0 als OpenSource-Software verfügbar und für interessierte Bibliotheken und Informationseinrichtungen nachnutzbar. ${ }^{16}$

Der Quellcode wird auf GitHub veröffentlicht und kann somit bei Bedarf an die eigenen Bedürfnisse angepasst werden. Bei der Installation müssen einige Daten wie

16 https://github.com/NTNM-Bib/Vidijo. 


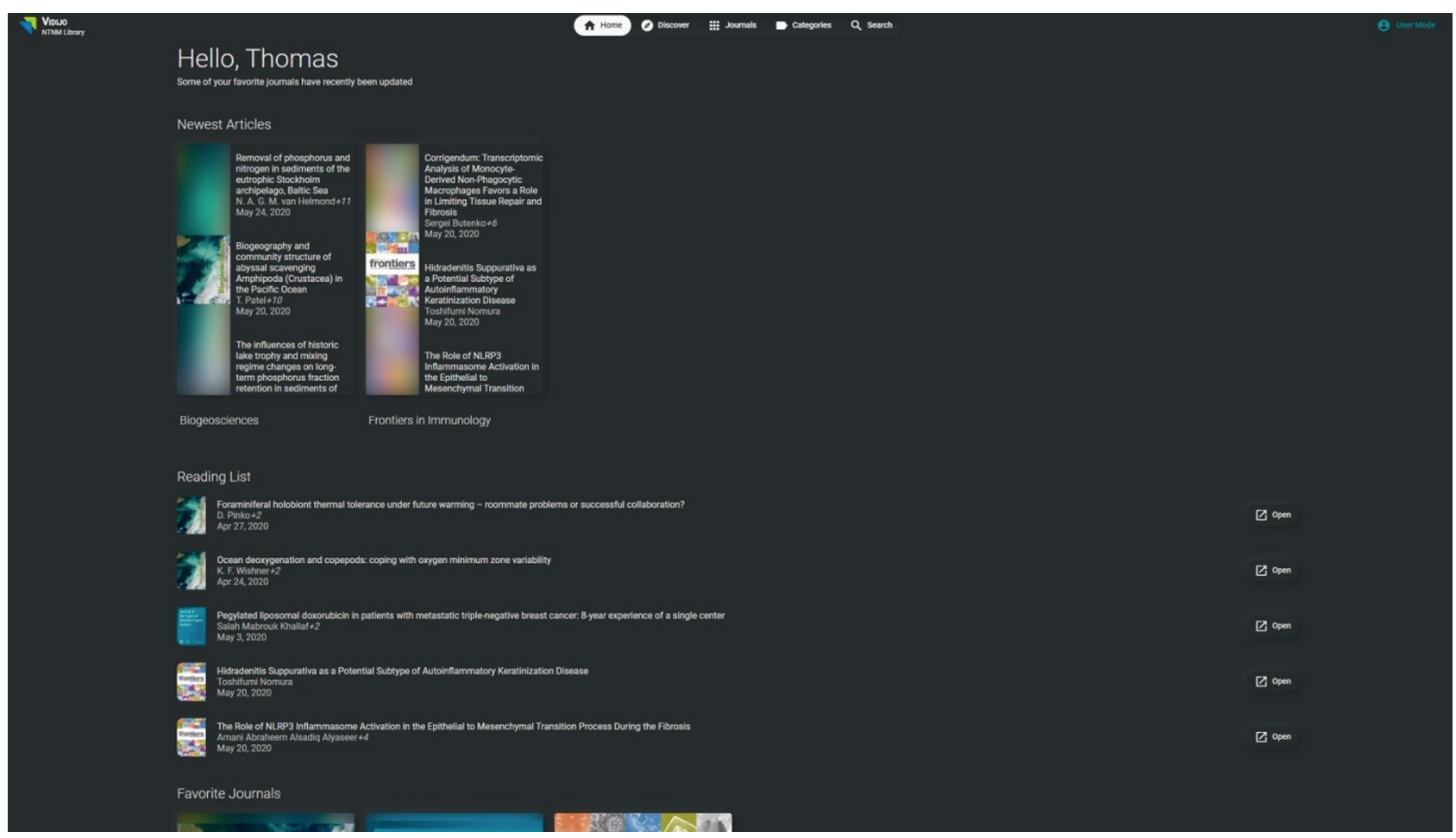

Abb. 3: Ein persönliches Konto bei VIDIJO

z. B. der Name der Einrichtung oder das eigene Logo angegeben werden; diese Daten werden verwendet, um z.B. das Aussehen der App anzupassen oder um die Erstellung von Nutzeraccounts $\mathrm{zu}$ ermöglichen. Nach erfolgreicher Einrichtung muss ein Administrator-Account erstellt werden, der automatisch die notwendigen Berechtigungen zum Hinzufügen von Journals, Editieren von Kategorien und mehr erhält. Alle folgenden Accounts erhalten lediglich normale Privilegien; weitere Administrator-Accounts können durch bereits existierende Administratoren angelegt werden, um die eigene VIDIJO-Installation gemeinsam zu verwalten.

Ein erster Workshop zum Projekt visOA, VIDIJO und Nachnutzung ${ }^{17}$ fand bereits am 27. November 2019 im INM - Leibniz-Institut für Neue Materialien statt. Unter den Teilnehmern befanden sich Mitarbeiter von Universitätsbibliotheken, Hochschulbibliotheken und Forschungsinstituten sowie eines Bundesamtes und einer kulturellen Stiftung. Die Bibliothek einer weiteren Leibniz-Einrichtung, Schloss Dagstuhl - Leibniz-Zentrum für Informatik, hat noch Ende November 2019 ihren OPAC (auf Basis eines anderen lokalen Bibliothekssystems) entsprechend erweitert und zeigt nun ebenfalls bei Open-Access-Publikationen ein Open-Access-Logo statt eines Coverbildes an.

17 https://visoa.leibniz-inm.de/nachnutzung.
Zuletzt hat Mitte April 2020 das Leibniz-Institut für Gewässerökologie und Binnenfischerei (IGB) sich am Beispiel des INM orientiert und kennzeichnet in den Publikationslisten auf seiner Instituts-Webseite die Open-Access-Publikationen ebenfalls mit einem Open-Access-Logo. Zudem finden Gespräche mit weiteren Interessenten über Kooperationen bei der Nachnutzung von VIDIJO statt. Es liegen darüber hinaus auch einige Anfragen vor, ob VIDIJO eventuell nicht nur Open-Access-Journals, sondern auch Closed-Access-Zeitschriften (also den gesamten elektronischen Zeitschriftenbestand einer Bibliothek) präsentieren könne. ${ }^{18}$

Nach der Freigabe der Version 1.0.0 als Open-SourceSoftware sollen weitere Workshops zur Nachnutzung angeboten werden.

Der Arbeitsvertrag des studentischen Mitarbeiters, der VIDIJO programmiert hat, wurde nach Projektende und dem Auslaufen der Fördermittel am 31.12.2019 vom INM noch bis Ende Februar 2021 verlängert, damit VIDIJO wei-

18 Das ist durchaus denkbar. Im Projekt visOA, in dem es um OpenAccess-Publikationen geht, wurde jedoch nur eine Schnittstelle zum DOAJ programmiert. Um auch Closed-Access-Zeitschriften anzeigen zu können, müssten entsprechende Schnittstellen zu anderen Quellen, wie z.B. JournalTOCs oder Crossref, programmiert werden. Im Idealfall könnten nachnutzende Institutionen die für ihre Bedarfe programmierten Schnittstellen auch anderen Einrichtungen der Nachnutzer-Community zur Verfügung stellen. 
terentwickelt werden und das bisherige Projektteam anderen Einrichtungen die Nachnutzung ermöglichen bzw. erleichtern kann.

Bei Interesse an einer Nachnutzung VIDIJOs in Ihrer Institution können Sie gern mit uns über bibliothek@leib niz-inm.de Kontakt aufnehmen.

\section{Literaturverzeichnis}

Goldschmitt, Regina (2015): JournalTouch: Open-Source-Tool für die digitale Zeitschriftenheftauslage. In: BIBLIOTHEK - Forschung und Praxis 39 (3), 342-49. DOI:10.1515/bfp-2015-0041.

Herb, Ulrich (2019a): Bibliography on criteria for assessing the quality of scientific publications. In: Zenodo. DOI:10.5281/zenodo.3366168.

Herb, Ulrich (2019b): Instructions for qualitative interviews on the qualitative perception of scientific publications. In: Zenodo. DOI:10.5281/zenodo.3482888.

Herb, Ulrich; Geith, Uwe (2020): Kriterien der qualitativen Bewertung wissenschaftlicher Publikationen. In: Information. Wissenschaft \& Praxis, 71 (2-3), 1-9. DOI:10.1515/iwp-2020-2074.

Kleiner, Eike (2014): Blended Shelf - Ein realitätsbasierter Ansatz zur Präsentation und Exploration von Bibliotheksbeständen. Wiesbaden: Dinges \& Frick (B.I.T.online INNOVATIV: 47).

Kleiner, Eike; Rädle, Roman; Reiterer, Harald (2013): Blended shelf: reality-based presentation and exploration of library collections. In: CHI '13 Extended Abstracts on Human Factors in Computing Systems (CHI EA '13). Association for Computing Machinery, New York, NY, USA, 577-82. DOI:10.1145/2468356.2468458.
Patel, Divyakumari (2019): Ein Vergleich von BrowZine, Paperboy und JournalTouch zur Präsentation von elektronischen Medien. Gekürzte Vers. Verfügbar unter https://depositonce.tu-berlin.d e/bitstream/11303/9215/4/patel_divyakumari.pdf.

Reiterer, Harald; Rädle, Roman; Butscher, Simon et al. (2016): Blended Library - neue Zugangswege zu den Inhalten wissenschaftlicher und öffentlicher Bibliotheken. In: BIBLIOTHEK Forschung und Praxis, 40 (1), 7-20. DOI:10.1515/bfp-2016-0010.

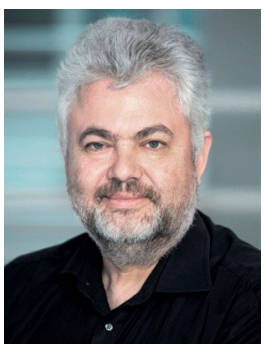

\author{
Uwe Geith \\ INM - Leibniz-Institut für Neue Materialien \\ $\mathrm{gGmbH}$ \\ NTNM-Bibliothek \\ Campus C6 2 \\ D-66123 Saarbrücken \\ uwe.geith@leibniz-inm.de \\ https://orcid.org/0000-0002-5654-9479
}

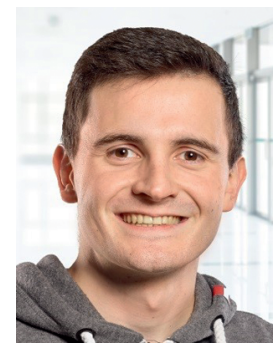

Thomas Kraß

INM - Leibniz-Institut für Neue Materialien gGmbH NTNM-Bibliothek

Campus $\mathrm{C} 62$

D-66123 Saarbrücken

krass@ntnm-bib.de 